\title{
Kernos
}

Revue internationale et pluridisciplinaire de religion grecque antique

18 | 2005

Varia

\section{La notion de retournement et l'agôn musical entre Apollon et Marsyas chez le ps.-Apollodore}

Interprétation d'un mythe

\section{Philippe Monbrun}

\section{(2) OpenEdition \\ Journals}

Édition électronique

URL : http://journals.openedition.org/kernos/1531

DOI : 10.4000/kernos. 1531

ISSN : 2034-7871

Éditeur

Centre international d'étude de la religion grecque antique

\section{Édition imprimée}

Date de publication : 1 janvier 2005

Pagination : 269-289

ISSN : 0776-3824

\section{Référence électronique}

Philippe Monbrun, «La notion de retournement et l'agôn musical entre Apollon et Marsyas chez le ps.Apollodore ", Kernos [En ligne], 18 | 2005, mis en ligne le 08 juillet 2011, consulté le 30 avril 2019. URL : http://journals.openedition.org/kernos/1531; DOI : 10.4000/kernos.1531 


\title{
La notion de retournement et l'agôn musical entre Apollon et Marsyas chez le ps.-Apollodore : interprétation d'un mythe
}

\begin{abstract}
Résumé : Dans la Bibliothèque (I, 4, 2), le pseudo-Apollodore raconte la joute musicale qui oppose Apollon au satyre Marsyas. Le dieu musicien l'emporte parce qu'il joue de sa cithare après l'avoir retournée, exécutant une manœuvre que son rival est incapable de reproduire avec l'aulos. Apollon fait ainsi de sa cithare un instrument palintone et palintrope, très proche de son arc réflexe, un instrument capable d'inverser sa position, mais aussi de renverser la situation au profit de celui qui l'utilise. Même "conduite de retournement » pour la lyre, dont la réversibilité efficace est redevable à la tortue ainsi qu'à Hermès et à son travail de luthier. La puissance de retournement des instruments à cordes apolliniens permet de jeter un éclairage nouveau sur la victoire d'Apollon et sur l'écorchement de Marsyas, tout en situant l'épisode dans le champ de la mètis. De l'instrument d'Apollon, retourné vers le bas, qui précipite Marsyas aux Enfers, à celui d'Orphée, tenu à l'endroit, qui en ramène Eurydice, se dessine la nature ambivalente de la lyre symétrique à celle de l'arc. Comme lui, la lyre est bien "signe de chute », de la vie vers la mort, ou «signe de remontée », de la mort vers la vie.
\end{abstract}

Abstract: The reversal's notion and the musical agôn between Apollon and Marsyas in ps.Apollodorus: interpretation of a myth. In The Library (I, 4, 2), the pseudo-Apollodorus reports how Apollo, the musician god, opposed to Marsyas the satyr, won the musical contest by playing his kithara upside down, a trick his opponent was quite unable to reproduce with his aulos. Thus, Apollo turned his kithara into a palintonos and palintropos instrument, very much like his reflex bow, an instrument, whose position could be reversed but which could also reverse the situation to the benefit of its user. The same reversal ability is true of the lyre, which could be played efficiently both ways thanks to the tortoise and to Hermes, the skilful lyremaker. The extraordinary capacity of Apollinian stringed instruments to be reversed throws a new light on both Apollo's victory and Marsyas's skinning, setting the episode in the mètis area. From Apollo's reversed lyre hurling Marsyas down into Hades to Orpheus's being played the right way, to bring back Euridyke from the underworld, emerges the ambivalent nature of the lyre in perfect symmetry with the bow's. Just like it, the lyre means the fall from life into death but it also represents the ascent from death into life.

\section{Quand un aulète défie un citharode}

L'agôn musical ${ }^{1}$ entre Apollon et Marsyas est le plus célèbre des concours musicaux de la mythologie grecque. À la cithare d'Apollon, le satyre phrygien oppose l'aulos, qu'A. Bélis définit comme un «instrument à vent à deux tuyaux, ébranlé par deux anches doubles »' Ni flûte, ni hautbois ou clarinette,

\footnotetext{
${ }^{1}$ Sur l'« esprit agonistique », « donnée fondamentale de la vie musicale, comme elle l'était de la culture et de la société grecques ", voir A. BéLIs, Les Musiciens dans l'Antiquité, Paris, Hachette Littératures, 1999 (La Vie quotidienne), p. 125-127, notamment.

${ }^{2}$ Ibid., p. 16.
} 
il n'a pas d'équivalent exact dans notre organologie. On connaît les circonstances qui ont conduit à cette joute, ainsi que son déroulement et son issue tragique pour Marsyas. Tout commence avec Athéna à laquelle la tradition la plus répandue, illustrée notamment par Pindare et Platon ${ }^{3}$, attribue l'invention de l'aulos et de l'aulétique. La déesse rejette l'instrument dès qu'elle voit les horribles traits de Gorgone que prend son visage quand elle en joue ${ }^{4}$. Marsyas, dont une autre tradition, attestée par Diodore et Athénée, fait l'inventeur de l'aulos ${ }^{5}$, recueille alors l'instrument délaissé par Athéna et ose défier Apollon, le dieu musicien, sur son propre terrain ${ }^{6}$. Mais l'bybris ne paie pas, comme le montre, par exemple, un cratère en cloche campanien, vers 360-330 (Fig. 1).

On voit Apollon, en habit de concours, qui vient de terminer sa prestation. Il est descendu du podium et semble détendre les cordes sur le joug de sa cithare. Nikè s'apprête à le couronner, alors qu'assis à ses pieds, Marsyas est vaincu, la tête appuyée sur la main gauche qui tient l'aulos reconnaissable à ses tuyaux rectilignes d'égale longueur. Artémis, tenant une lance, un chien auprès d'elle, assiste à la victoire de son frère. La suite de l'épisode est représentée sur un médaillon gallo-romain de la seconde moitié du i ${ }^{\text {er }}$ siècle (Fig. 2) : sur la droite de l'image, accoudé à sa cithare, le dieu vainqueur condamne Marsyas à être écorché vif. À ses pieds, Olympos, ici fils de Marsyas, implore en vain la clémence. En face d'Apollon, le drame est seulement suggéré par le bourreau Skythès apprêtant ses lames pendant que son aide hisse la victime pour la suspendre à la branche d'un arbre.

\footnotetext{
3 Respectivement, Pythiques XII, 6-8, 19-22 et Politique VIII, 6, 13. Voir aussi Diodore, V, 73, 7-8; Ovide, Fastes, 695-698, 709 et Hygin, Fables, 165, 1.

4 Ainsi, Platon, Politique VIII, 6, 14; Apollodore, I, 4, 2; Ovide, Fastes, 699-702; Plutarque, Du contrôle de la colère, 6 et Hygin, Fables, 165, 2. Sur le rejet de l'aulos par Athéna et l'interprétation des mimétismes sonore et visuel de la déesse avec Gorgô, il faut voir les analyses de Z. PAPAdopoulou et V. PIRENNe-Delforge, «Inventer et réinventer l'aulos : autour de la XII Pythique de Pindare", in P. BRulé, Ch. VENDrIEs (éds), Chanter les dieux. Musique et religion dans l'Antiquité grecque et romaine, Actes du colloque des 16, 17 et 18 décembre 1999, Rennes et Lorient, P.U.R., 2001, p. 38-47.

Respectivement, III, 58, 3 et IV, 18 a. Invention attribuée à Hyagnis, le père et le maître de Marsyas, par Apulée, Florides III, 1-5.

Le concours musical et le châtiment infligé par Apollon ont été popularisés par la littérature et l'iconographie. Pour les sources littéraires, voir A. WEIss, « Marsyas I », LIMC VI.1 (1992), p. 367. On peut retenir, pour l'essentiel, Hérodote, VII, 26; Xénophon, Anabase I, 2, 8; Apollodore, I, 4, 2; Diodore, III, 59, 1-5; Ovide, Métam. VI, 382-400 et Fastes, 703-708; Hygin, Fables, 165, 3-5; Apulée, Florides, III, 6-14; Philostrate le Jeune, Images, 2, 1-4 et Nonnos de Panopolis, Dionysiaques XIX, 316-327. Pour les documents figurés, il faut voir le catalogue du LIMC VI.2 (1992), à " Marsyas I », pl. 185-193 et les commentaires de WEISs, ibidem (VI.1), p. 370378. Je renvoie aussi aux commentaires de L. KAHIL sur les vases attiques $n^{\circ} 1420-1426$ et 1432 , sur les vases italiotes $n^{\circ} 1427-1430$, ainsi que sur la mosaïque n 1431 dans « Artémis », LIMC II.1 (1984), p. 733-735. Sur le châtiment de Marsyas, voir encore M. HaLm-Tisserant, Réalités et imaginaire des supplices en Grèce ancienne, Paris, Les Belles Lettres, 1998 (Coll. Études anciennes, 125), p. 56-60 et pl. 6.

Sur le Scythe coupeur de tête, tailleur de scalp, écorcheur, tanneur et autre travailleur de peau humaine, voir Fr. HARTOG, Le miroir d'Hérodote. Essai sur la représentation de l'autre, Paris,
} 
Fig. 1

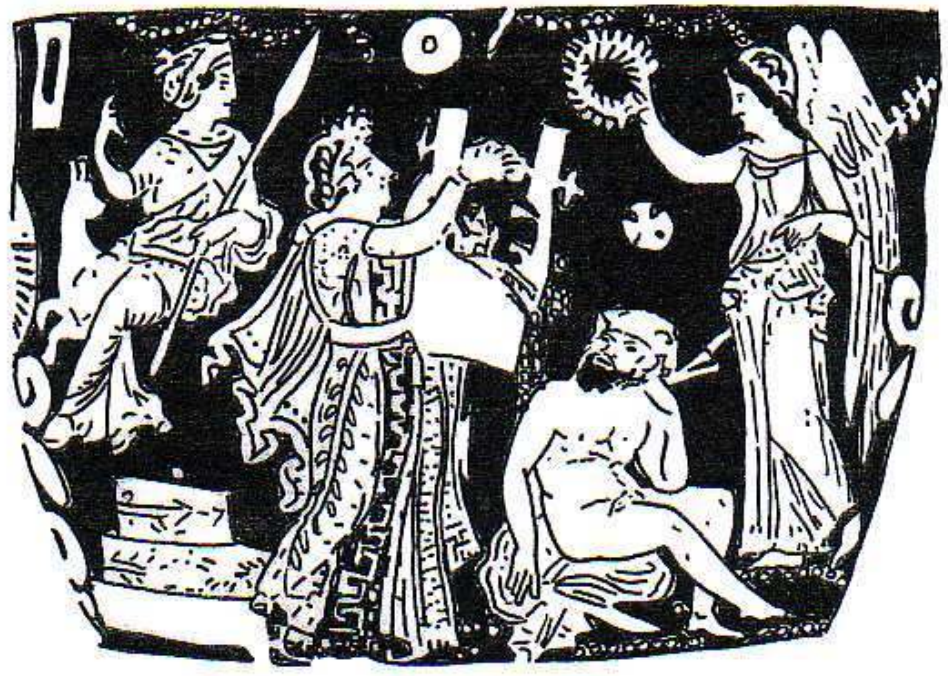

Ce sont les conditions de la victoire d'Apollon qui m'intéressent au premier chef. Selon la tradition la plus communément reçue, Apollon l'emporta parce que lui seul pouvait chanter tout en jouant de son instrument. Cette version a le mérite de situer l'agôn entre Apollon et Marsyas dans le cadre des vicissitudes de la vie musicale grecque. C'est Diodore qui en donne le récit le plus exemplaire (III, 59, 2-3):

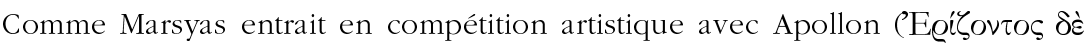

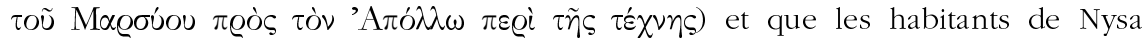
avaient été pris comme juges, Apollon, en premier, joua de la cithare sans accom-

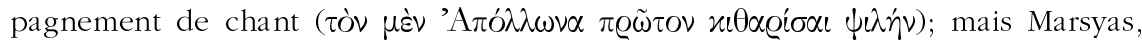

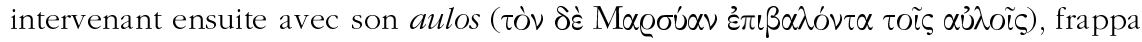

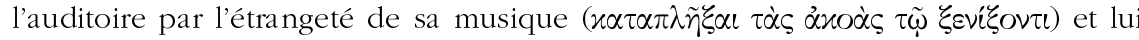
parut l'emporter de beaucoup sur le premier concurrent par ses qualités mélodieu-

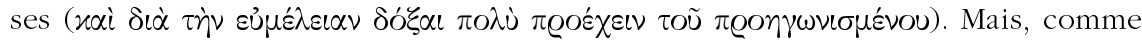
ils étaient convenus de montrer alternativement ( $\left.\pi \alpha Q ’ \alpha^{\prime} \lambda \lambda \eta \lambda \alpha\right)$ leur art aux juges,

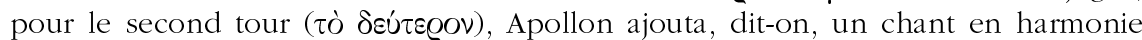

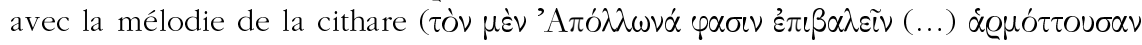

Gallimard, 1980, p. 171-179; V. SCHILTZ, Les Scythes et les nomades des steppes. VIII siècle avant JC. - $I^{e r}$ siècle après J.-C., Paris, Gallimard, 1994 (L'Univers des formes), p. 406, 417-434 et I. LeBeDYNSKI, Les Scythes. La civilisation nomade des steppes (VII'-III siècles av. J.-C.), Paris, Errance, 2001, p. 174-175. Comme en témoignent Hérodote (IV, 64-65) et Athénée (IX, 410c), cette réputation est, chez les Grecs, inséparable de l'image du Scythe, à tel point que le verbe skythizô, "boire immodérément (comme un Scythe)», "parler scythe», peut aussi signifier "se raser la tête (à la Scythe) », d'après l'habitude qu'avaient les Scythes de scalper leurs prisonniers. Cf. Euripide, Electre, 241. Je renvoie au Greek-English Lexicon (with a revised Supplement) de $\mathrm{H}$. G. LidDELL, J. ScOtT et H.S. Jones (cité LSD), Oxford, Clarendon Press, 1996, sous $\sum x u ́ \theta \alpha i v \alpha$ ainsi que sous les composés $\dot{\alpha} \pi 0 \sigma \varkappa u \theta i \zeta \omega$ et $\pi \varepsilon \varrho \iota \sigma \varkappa u i \zeta \omega$. Sur les usages de langue dans ce registre, voir particulièrement HARTOG, ibidem, p. 173. 
$\tau \tilde{\varphi} \mu \dot{\varepsilon} \lambda \varepsilon \imath \tau \tilde{\eta} \subseteq \varkappa \theta \dot{\alpha} \varrho \alpha \varsigma \varphi \hat{\phi} \delta \dot{\eta} \nu)$ et acquit ainsi une plus grande faveur que celle que

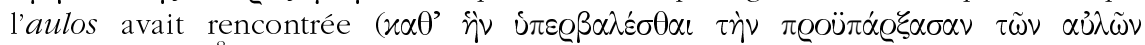
$\dot{\alpha} \pi O \delta \circ \chi \dot{n} \nu)(\ldots)^{8}$.

Indigné, Marsyas conteste alors la validité de la performance en faisant valoir que le concours devait uniquement porter sur l'art instrumental (III, 59, 3). Apollon réplique que tous deux utilisent leurs mains et leurs bouches (III, 59, 4) et emporte l'avis des juges (III, 59, 5) :

Et comme les auditeurs avaient jugé que les paroles d'Apollon étaient les plus justes, l'on fit une nouvelle confrontation de leurs arts; Marsyas eut le dessous (M $\propto \varrho \sigma u ́ \alpha \nu \lambda \varepsilon\llcorner\varphi \theta \tilde{r} \nu \alpha \iota)$, et Apollon, que la querelle avait passablement irrité, écorcha

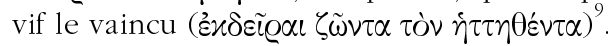

Fig. 2

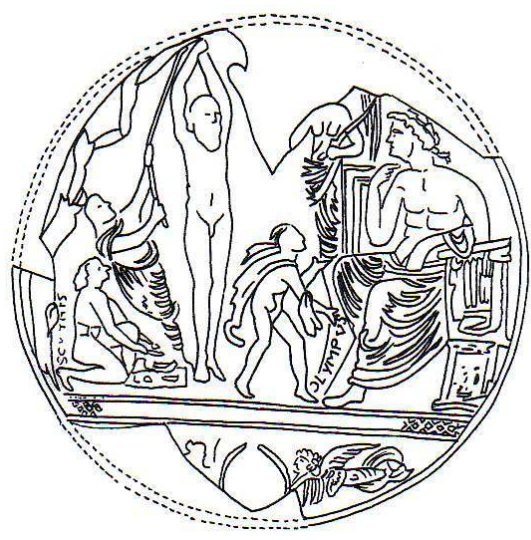

On retrouve, dans cette version du concours, la supériorité qui était reconnue à la citharodie parce qu'elle exigeait de chanter et de jouer de la cithare en même temps. Comme le souligne A. Bélis, "le monde antique ne douta jamais que la citharodie était la plus belle et la plus difficile des spécialités musicales ${ }^{10}$. Elle combinait, en effet, au prix d'un travail acharné, la maîtrise simultanée de la citharistique, strictement instrumentale, et de la technique du chant. Dans De l'Institution oratoire (I, 12, 3), le rhéteur Quintilien apprécie en connaisseur l'exploit du citharode : il doit surveiller sa mémoire et marquer le rythme du pied; il doit se concentrer à la fois sur son chant et sur les jeux différents et complémentaires de ses deux mains pour marier parfaitement les accents de sa voix et de sa cithare. On peut ajouter aux exigences relevées par Quintilien que, lors des récitals et des concours panhelléniques ou régionaux, les citharodes étaient aussi jugés sur la qualité de leur expressivité et de leur gestuelle. Aussi, que Marsyas, par ses talents d'aulète, ait été prêt de l'emporter sur Apollon cithariste, pourquoi pas. L'aulos est réputé être l'instrument le plus polyvalent de l'organologie grecque, le plus capable d'exprimer tous les sentiments et d'accompagner toutes les circonstances. Mais, face à Apollon citharode, le satyre aulète ne faisait plus le poids. Virtuose, dans le même temps, du chant

\footnotetext{
${ }^{8}$ Texte établi et traduit par B. BOMmELAER, Collection des Universités de France (citée CUF), Paris, Les Belles Lettres, 1989. J'ai préféré aulos à « double flûte ».

9 Traduction de B. BOMMELAER, ibidem.

10 BÉLIs, o.c. (n. 1), p. 99 et p. 185 : «Pratiquée par les musiciens les plus chevronnés, elle suscita une admiration sans bornes dont les textes grecs et latins portent le constant témoignage. »
} 
et de la cithare, le dieu se révèle un musicien plus accompli que Marsyas. En effet, souffler dans les tuyaux de l'aulos tout en chantant est une prouesse qui ne se rencontre guère qu'à l'état de non-sens dans les versions grecque ou latine. Il existait bien une discipline combinant le chant et la musique de l'aulos. C'est l'aulodie, où un chanteur, appelé aulode, était accompagné par un aulète, mais, par la force des choses, elle nécessitait deux exécutants. Un Marsyas aulode n'aurait donc pu concourir avec un Apollon citharode et même s'il l'avait pu, il n'aurait eu aucune chance de l'emporter, l'aulodie étant considérée comme une discipline vocale de second choix par rapport à la citharodie qui couronnait l'ensemble des disciplines musicales. À bien des égards, en effet, le cithariste et l'aulode apparaissent comme des musiciens incomplets, chacun d'eux ne maîtrisant qu'une moitié de la citharodie ${ }^{11}$. À la suite des textes anciens, A. Bélis relève une autre «différence capitale » entre les deux disciplines : alors que l'aulos « couvre facilement la voix », «l'accompagnement par la seule cithare ne masquait aucune erreur», ce qui rendait l'exercice particulièrement difficile ${ }^{12}$. Rien de surprenant, donc, à ce que les récompenses les plus importantes aient été décernées aux citharodes ${ }^{13}$.

Le rejet de l'aulos par Athéna, la défaite et la condamnation de Marsyas, trouvent aussi leur place dans le cadre des contestations suscitées par l'aulos au début de l'époque classique, ainsi que l'ont mis en évidence Z. Papadopoulou et V. Pirenne-Delforge dans un article consacré à la XII Pythique de Pindare $^{14}$ : capable d'imiter au mieux la voix humaine tout en étant à la fois fonctionnellement indisponible pour le chant et assez puissant pour le couvrir, l'aulos tend à marginaliser le logos. Dans les chœurs dédiés à Apollon, sa musique doit rester au second plan, au service de la parole chantée que préfère le dieu. Au v siècle, la cité démocratique d'Athènes veille aussi à défendre la primauté du chœur civique, du chant et de la parole, menacée par l'aulos et la musique en quelque sorte politiquement incorrecte qu'il représente. Qu'est-ce donc que la défaite de l'aulos dans l'agôn entre Apollon et Marsyas tel que le raconte Diodore ? Elle apparait, d'une part, comme la très apollinienne victoire du chant, accompagné et non masqué par l'instrument, et d'autre part, comme la très démocratique victoire de la musique

\footnotetext{
11 Deux exemples suffisent pour illustrer la primauté de cette dernière. Élien, dans l'Histoire Variée (IV, 2) rapporte la saillie d'un cithariste reprochant à un citharode d'être petit dans un «art majeur », alors que lui était grand dans un «art mineur » : malgré le trait d'esprit, la hiérarchie est affirmée et le citharode n'en reste pas moins supérieur par ses talents de chanteur. Cicéron, dans le Pro Murena (XIII, 29), rapporte l'expression proverbiale grecque selon laquelle on devenait aulode faute d'avoir pu être citharode: ici c'est le chanteur qui n'est pas suffisamment bon cithariste pour faire un citharode.

12 BÉLIS, o.c. (n. 1), p. 184-185.

13 Ainsi, lors des épreuves musicales des Panathénées, en 380 avant J.-C., le prix du deuxième citharode est largement supérieur à celui du cithariste vainqueur, tandis que le prix du cinquième et dernier citharode classé est égal à celui du premier aulode. Voir BÉLIS, o.c. (n. 1), p. 146.

14 Papadopoulou, V. Pirenne-Delforge, l.c. (n. 4), p. 53-55. Sur l'incompatibilité entre l'aulos et la parole, au contraire de la cithare d'Apollon qui permet le jeu et le chant à un seul et même interprète, voir B. LECLERCQ-NeveU, « Marsyas, le martyr de l'aulos », Metis 4 (1989), p. 261.
} 
chorale, expression du corps civique, sur l'bybris du soliste virtuose incompatible avec la formation morale du citoyen ${ }^{15}$.

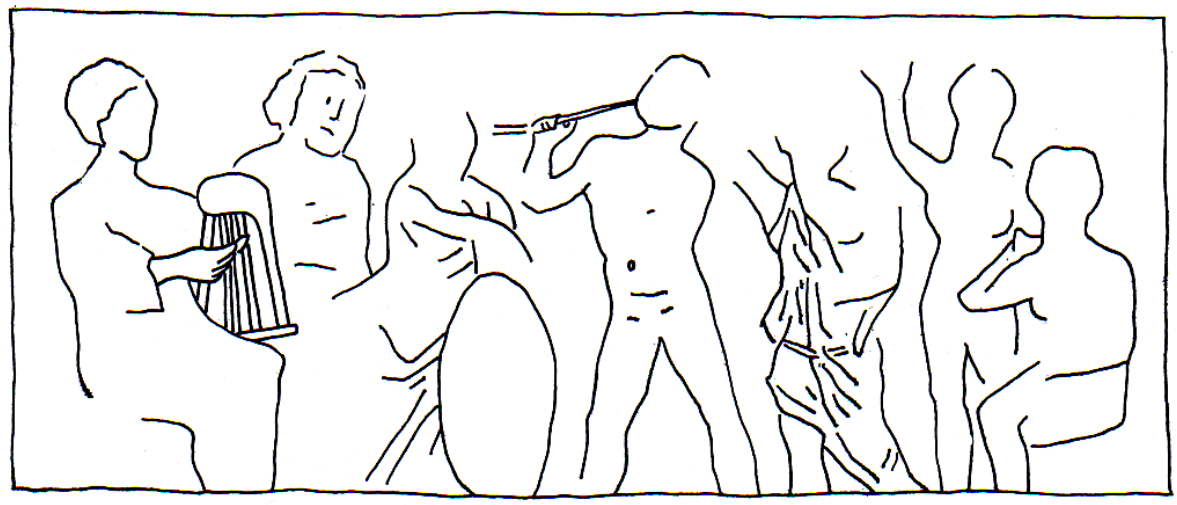

Fig. 3

\section{Comment Apollon retourne la cithare à son profit}

J'en resterai là sur ces réflexions. Il est temps de considérer l'autre raison invoquée pour la victoire d'Apollon, notamment par l'auteur de cette compilation mythologique qu'est la Bibliothèque. Le ps.-Apollodore, à qui l'on doit la première narration complète des tragiques mésaventures de Marsyas, raconte comment Apollon gagne le concours en exécutant une manœuvre de retournement que son adversaire est dans l'impossibilité de reproduire (I, 4, 2) :

Apollon tua aussi Marsyas, le fils d'Olympos. Celui-ci, en effet, trouva l'aulos qu'Athéna avait jeté parce qu'il enlaidissait son visage et il engagea une joute

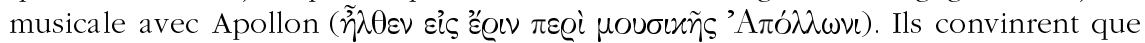

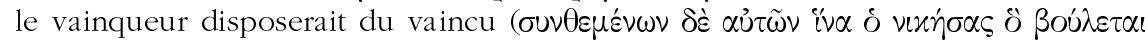

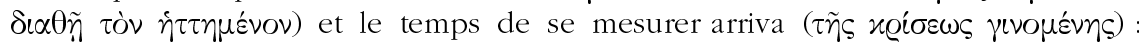

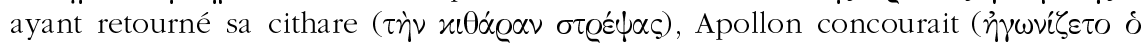

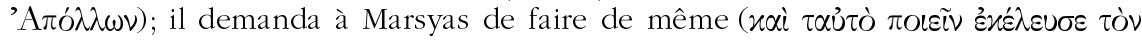

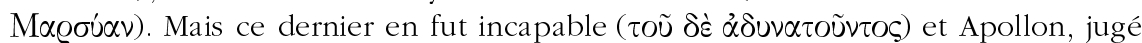

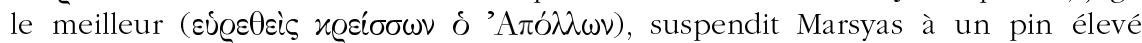

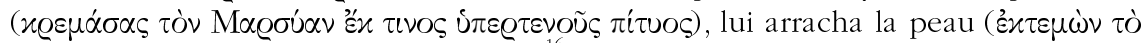

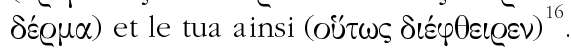

Si Apollon est vainqueur, c'est bien parce qu'il est capable de jouer de sa

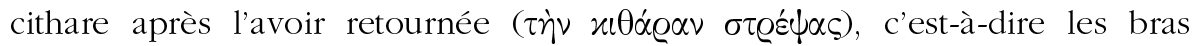
orientés vers le bas et la caisse de résonance vers le haut. J. G. Frazer l'a bien compris qui traduit, en 1921 dans la Loeb Classical Library, « Apollo turned his lyre upside down in the competition », c'est-à-dire qu'Apollon «tourna à

\footnotetext{
15 Pour Z. Papadopoulou, V. Pirenne-Delforge (l.c., p. 55), «l'agôn entre Apollon et Marsyas est essentiellement une joute entre logos et performance musicale d'un virtuose ».

Je propose ici ma traduction. Le texte grec est celui sur lequel s'appuie la traduction de J. G. Frazer dans la Loeb Classical Library (citée LCL), 1921.
} 
l'envers » ou «retourna » son instrument pour en jouer. L'anthropologue britannique appuie sa lecture sur la comparaison avec les passages parallèles d'Hygin et du Second Mythographe du Vatican ${ }^{17}$. Dans la fable 165, Hygin vient de raconter comment Marsyas trouve l'aulos dont s'est débarrassée Athéna, s'entraîne à en jouer et provoque en duel Apollon et sa cithare (1-3) Il continue (4-5) :

Quand Apollon fut arrivé, ils prirent les Muses pour juges, et comme Marsyas repartait vainqueur (et cum iam Marsyas inde victor discederet), Apollon retournait la cithare (Apollo citharam versabat) et il fit entendre les mêmes accents (idemque sonus erat). Marsyas fut incapable d'en faire autant avec l'aulos (quod Marsyas tibiis facere non potuit). Ainsi, Apollon lia Marsyas vaincu à un arbre et le remit à un Scythe (Itaque Apollo Marsyan ad arborem religatum Scythae tradidit) qui le dépeça membre à membre (qui eum membratim separavit) ${ }^{18}$.

À l'expression Apollo citharam versabat, répond celle du Mythographe du Vatican (II, 115) : invertit citharam et canere coepit, « il retourna la cithare et se mit à jouer » ${ }^{19}$.

Invoquant les trois mêmes textes, Burckhardt, auteur, en 1930, de l'article sur le satyre Marsyas dans la Real-Encyclopädie, comprend lui aussi que, «pendant le jeu» (beim Spiel), Apollon a «retourné » (« umgekehrt») sa cithare et a demandé à Marsyas de l'imiter ${ }^{20}$. Même lecture de B. LeclercqNeveu dans la revue Mètis, en 1989 : «Apollon prit sa lyre à l'envers pour jouer et défia moqueusement son adversaire d'en faire autant ${ }^{21}$. Marsyas ne put évidemment pas relever le défi de jouer de l'aulos à l'envers : son instrument à vent et à anches est, par essence, un instrument "à sens unique » qui rend le chant impossible et interdit tout autant la réversibilité dans le jeu ${ }^{22}$. Cette joute insolite est illustrée par une plaque de grès en relief, provenant d'un monument funéraire romain. Découverte à Bierbach, en Allemagne, et publiée en 1892 dans la Westdeutsche Zeitschrift (XI, p. 88-99), cette pièce est conservée au Musée historique du Palatinat de Speyer. Burckhardt la présente comme l'unique témoin, dans tout l'art antique, du mythe d'Apollon et Marsyas selon la version qu'en donnent Apollodore et Hygin. Il commente, en usant du même terme que plus haut : "Apollo hält die Kithara umgekehrt », "Apollon tient la cithare retournée »". Même interprétation d'A. Weiss qui, dans le catalogue du LIMC, à «Marsyas I », range le document dans la section consacrée au concours avec Apollon. Il occupe seul la rubrique «Apollo

17 Ibidem, note 1 ad loc.

18 Traduction, légèrement modifiée, de J.-Y. BORIAUd, CUF, 1997.

19 J'ai traduit le passage sur le texte latin donné par J.G. FrAZER, o.c., note 1 ad loc. La suite précise que Marsyas retourna lui aussi son aulos, mais sans pouvoir égaler Apollon : Inversis autem tibiis, quum se Marsya Apollini aequiparare nequiret.

20 BurCKHARDT, «Marsyas (6) », RE XIV (1930), col. 1991, 1. 21-26.

21 LECLERCQ-Neveu, l.c. (n. 14), p. 258 et p. 261 : «Marsyas est mis en difficulté parce qu'il ne peut imiter Apollon qui se met à jouer en tenant sa lyre à l'envers. »

${ }^{22}$ Sur le caractère non-réversible de l'aulos, voir LECLERCQ-NEvEU, ibid., p. 261-262.

23 BurckHARDT, l.c. (n. 20), col. 1995, 1. 2-9. 
exploits an advantage?» et A. Weiss commente ainsi : "Apollo plays the inverted cithara while M[arsyas] plays the flutes » ${ }^{24}$. Observons donc cette plaque en relief (Fig. 3).

On voit, malgré l'usure de la pierre, Apollon assis, sur la gauche, qui tient non pas une cithare, précisément, mais une lyre, bien reconnaissable à sa silhouette. L'instrument est retourné, la caisse de résonance en forme de carapace de tortue, vers le haut, les bras et le joug qui les réunit, vers le bas, appuyés sur la cuisse gauche du dieu. Apollon est en train de jouer comme le montre sa main droite, armée du plectre, sur le plan des cordes. Devant lui, vers la droite, un homme barbu et Athéna, accoudée à son bouclier. On reconnaît, au milieu de la scène, Marsyas jouant de l'aulos, et derrière lui, le bras droit levé, Nikè, suivie de deux personnages féminins, l'un debout, l'autre assis, peut-être les Muses du jury. Une telle représentation de la lyre tenue à l'envers, est suffisamment rare pour que je m'autorise à la rapprocher d'un type de statuettes votives en terre cuite des $\mathrm{V}^{\mathrm{e}}$ et $\mathrm{IV}^{\mathrm{e}}$ siècles, provenant de Véies et de Faléries et conservées à la Villa Giulia : elles figurent Aplu, l'Apollon étrusque, debout et nu, tenant sa lyre retournée, les bras vers le $\mathrm{sol}^{25}$. On retrouve la même position de la lyre sur un fragment du $\mathrm{V}^{\mathrm{e}}$ siècle trouvé dans le sanctuaire du Portonaccio, à Véies (Fig. 4, ci-dessous).

I. Krauskopf qui présente ces pièces dans le LIMC, note l'inversion de la lyre sans commentaire particulier ${ }^{26}$ et je me contenterai moi-même de les livrer à la sagacité du lecteur. Je continuerais volontiers mon chemin si J.G. Landels, dans un des derniers ouvrages généraux parus sur la musique dans l'Antiquité, ne contestait la lecture même du retournement de l'instrument apollinien dans la version du ps.-Apollodore. S'interrogeant sur la signification du terme strepsas dans l'ex-

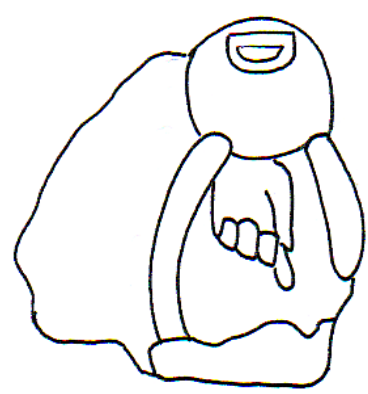

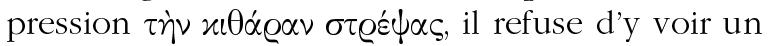
renversement de la cithare, arguant du fait que, s'il en est vraiment ainsi, rien n'empêche Marsyas de jouer lui aussi de l'aulos « upside-down », dit-il, " en se couchant sur le dos, comme le font souvent les satyres» («by lying on his back, as satyrs often do ») ${ }^{27}$. L'argument, à vrai dire, ne me paraît pas du tout convaincant.

Si Marsyas joue allongé sur le dos, à la façon de ce satyre (Fig. 5) assurant la partie musicale d'un symposion présidé par Dionysos, sur une amphore attique, vers 490, ce n'est pas l'aulos qui est retourné ou renversé : seul l'aulète a modifié sa position. Les anches de l'instrument restent dans la bouche du joueur tandis que le souffle et l'«air»musical sont toujours propulsés dans la même direction à travers les tuyaux.

24 WeIss, l.c. (n. 6), p. 372.

25 Voir I. KRausKopf, « Apollon / Aplu », LIMC II.2 (1984), fig. 90, p. 295.

${ }^{26}$ Ead., LIMC II.1 (1984), fig. 89-90, p. 348.

27 J.G. LANDELS, Music in Ancient Greece and Rome, Londres, Routledge, 2001, p. 156. 
Fig. 5

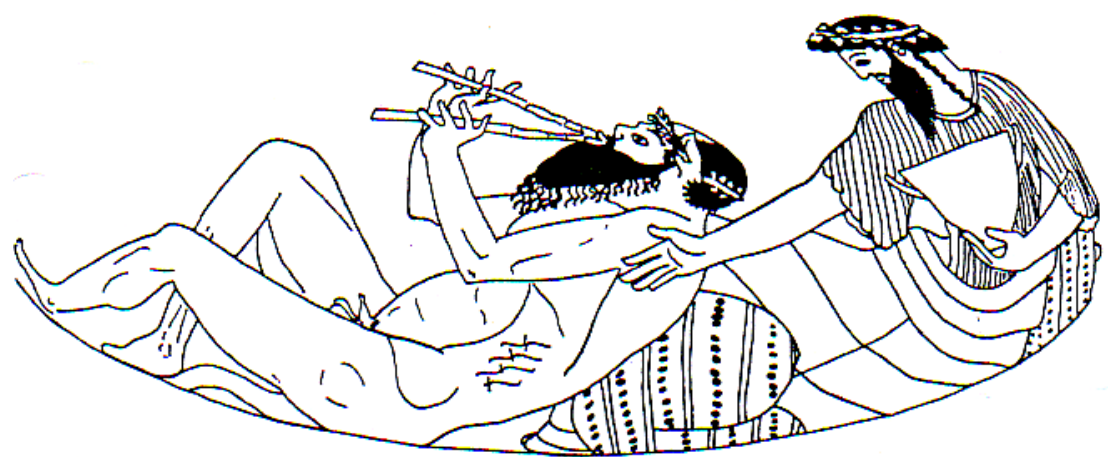

Pour que cette interprétation soit valable et que cette technique instrumentale puisse être une imitation de celle d'Apollon, il aurait fallu que le dieu ait joué de la cithare la tête en bas et les pieds en haut, en faisant le poirier ${ }^{28}$ ! Notons que même dans ce cas de figure aussi improbable qu'acrobatique, la cithare serait toujours tenue dans le bon sens et seul le cithariste, comme le satyre jouant couché, aurait une position renversée ou inversée. Il faut donc, me semble-t-il, écarter la solution que propose J.G. Landels : Apollon a bel et bien retourné l'instrument à cordes et a réussi à en jouer et, de là, on comprend pourquoi le dieu surclasse Marsyas qui ne peut retourner son aulos et transformer en embouchure les extrémités des deux tuyaux. En l'absence d'une explication suffisamment satisfaisante à ses yeux ${ }^{29}$, J.G. Landels fustige celle qui est, à mon sens, la seule possible :

Si cela signifie qu'on exigeait de Marsyas qu'il souffle dans son instrument dans le mauvais sens ( to blow the wrong way down his instrument»), cela ravale l'histoire toute entière au rang d'une ineptie stupide (" to fatuous nonsense »), et retire à Apollon tout mérite pour une victoire remportée grâce à une tricherie éhontée (« by a piece of blatant cheating ») .

28 C'est pourtant ce que semble imaginer G. NICOLE, auteur en 1911 de l'article « Satyri, sileni » dans le Dictionnaire des antiquités grecques et romaines (Ch. DAREMBERG, Éd. SAGLIO, Éd. POTTIER (éds), IV, 2, p. 1100), quand il écrit, en renvoyant à la fable d'Hygin, qu'Apollon exige de Marsyas qu'il joue « comme lui un même air sur l'instrument, debout puis renversé ».

29 LANDELS, o.c. (n. 27), n. 24, p. 285 : en quête d'une solution, l'auteur relève que certaines représentations montrent Apollon tenant son instrument figuré de dos, mais pour faire aussitôt le constat suivant : «Je ne vois pas comment ceci pourrait avoir le moindre rapport avec l'instrument de Marsyas. » En effet. Lors de la discussion qui suivit cette communication, P. Ellinger émit l'hypothèse qu'Apollon ait pu jouer de sa cithare en la tenant non pas retournée, bras vers le bas, caisse vers le haut, mais après l'avoir faite pivoter, le dos de l'instrument vers lui et le plan des cordes vers l'extérieur. C'est le seul autre retournement envisageable, mais il a contre lui la tenue de jeu sans ambiguïté d'Apollon sur la plaque en relief de la figure 3 et il remet en question la nécessaire symétrie avec Marsyas. L'impossible réversibilité de l'aulos et la défaite de Marsyas ne se comprennent que si le satyre échoue à jouer après avoir interverti les extrémités de son instrument, en réponse à Apollon qui a inversé le haut et le bas de sa cithare.

30 LANDELS, O.C. (n. 27), p. 156-157. 
Pour la même raison, B. Leclercq-Neveu qui, nous l'avons vu, comprend qu'Apollon joue de sa cithare après l'avoir renversée, porte un jugement de valeur similaire sur l'honnêteté d'Apollon : «Aussi la victoire d'Apollon paraîtelle bien spécieuse, et sa mauvaise foi criante »" Il est vrai qu'en tournant son instrument en sens inverse, Apollon berne effectivement Marsyas et ne brille guère par son fair-play. Mais pourquoi Apollon devrait-il avoir une moralité au-dessus de tout soupçon ? Et à quoi sert de déplorer le verdict final ou la rouerie du dieu? C'est faire un mauvais procès sinon à Apollon luimême, du moins à l'intelligence rusée, car c'est bien, me semble-t-il, de mètis qu'il s'agit et de l'ambiguité de la victoire qu'elle procure. M. Detienne et J.-P. Vernant ont bien posé le problème dès le début de leur livre sur les ruses de l'intelligence :

Par certains aspects, la mètis s'oriente du côté de la ruse déloyale, du mensonge perfide, de la traîtrise, armes méprisées des femmes et des lâches. Mais par d'autres elle apparaît plus précieuse que la force; elle est en quelque sorte l'arme absolue, la seule qui ait pouvoir d'assurer en toute circonstance, et quelles que soient les conditions de la lutte, la victoire et la domination sur autrui ${ }^{32}$.

De fait, si Apollon ne joue pas vraiment franc-jeu avec un adversaire qui, il faut le noter, a pris l'initiative de l'affronter sur un "terrain » où tous deux excellent, le dieu ne l'emporte pas moins en accomplissant un véritable tour de force, un exploit unique dans le domaine musical et à la seule portée du maître-musicien qu'il est ${ }^{33}$. Apollon recourt à la réversibilité efficace de son instrument de musique, et cette manœuvre plus ou moins frauduleuse est «l'arme absolue» qui lui permet de se sortir d'affaire face à un concurrent redoutable.

\section{Renversements de position et retournements de situation}

Ce sont les recherches que je mène sur et autour d'Apollon qui me conduisent à situer cette version du concours entre le dieu musicien et Marsyas dans le champ de la mètis. La confrontation du discours mythique sur le dieu et du discours en paroles et en images tenu par les Grecs sur ses différents champs d'activités, laisse apparaître combien l'arc, la lyre - le terme regroupe ici la phorminx, la kithara et la lyre proprement dite, la chelys des poètes - et le palmier-dattier, si chers à Apollon, intéressent cette forme particulière d'intelligence qui traverse la pensée grecque. M. Detienne et J.-P. Vernant ont mis en évidence combien la «conduite de retournement », ainsi qu'ils la nomment ${ }^{34}$, est une qualité fondamentale de la mètis. Maîtrisée par Hermès inversant le sens de sa marche dans l'Hymne bomérique qui lui est

\footnotetext{
31 LECLERCQ-NEVEU, l.c. (n. 14)., p. 259

32 M. Detienne, J.-P. Vernant, Les ruses de l'intelligence. La mètis des Grecs, Paris, Flammarion, 1974 , p. 20.

33 Cithariste et lyriste, A. Bélis m'a confirmé qu'il paraît impossible de jouer de la cithare ou de la lyre en la tenant renversée.

34 DETIENNE - VERNANT, o.c. (n. 32), p. 41, à propos du comportement du renard
} 
consacré, ainsi que par de nombreux "pensionnaires » du bestiaire de la mètis, cette qualité culmine chez le renard auquel les Grecs prêtent la faculté de se retourner brusquement pour repousser l'attaque de l'aigle. Dans la quatrième Isthmique, Pindare lui compare le pancratiste Mélissos de Thèbes qui, à première vue, ne paye guère de mine : tous deux, par un renversement du corps, échappent à la prise de leurs adversaires. Mélissos se laisse choir sur le dos pour esquiver l'assaut de son opposant, comme le renard se retourne pour bloquer l'attaque du prédateur ${ }^{35}$. Celui qui passait pour battu retourne donc la situation à son profit : renversé sur le dos, apparemment en situation d'infériorité, il surprend le vainqueur présumé et prend le dessus sur lui. Efficace, le renversement de position est aussi un retournement de situation grâce auquel le plus faible ou le plus léger arrive à l'emporter sur plus fort ou plus lourd que lui, ce qui est un paradoxe caractéristique de la mètis.

\section{Le palmier-dattier}

Cette victorieuse «conduite de retournement» se retrouve chez l'arbre de la «nativité » délienne d'Apollon et d'Artémis. Comme le rapporte Théophraste dans les Recherches sur les plantes (V, 6, 1),

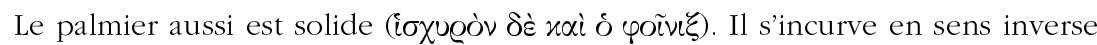

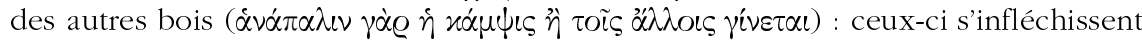

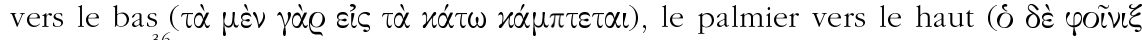

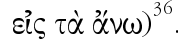

Le palmier-dattier (phoinix) était réputé se courber "en sens inverse» (anapalin) des autres bois et c'est un topos que l'on retrouve chez de nombreux auteurs ${ }^{37}$ : au lieu de s'infléchir sous le poids, il se voûte, comme une étagère qui se bomberait sous des livres, triomphant ainsi des plus lourdes

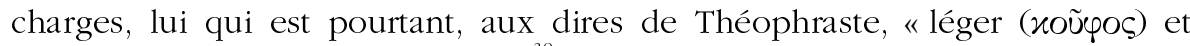

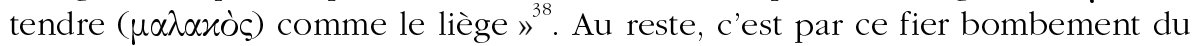
bois de palmier, par ce comportement spécifique qui lui permet de résister victorieusement à une force contraire, que Plutarque, dans les Propos de table (VIII, 4, 1-5), et Aulu-Gelle, dans les Nuits Attiques (III, 6, 3), expliquent pourquoi une palme est offerte à l'athlète vainqueur ${ }^{39}$. Cette curieuse propriété du

\footnotetext{
35 Isthm. IV, 45-51. Élien (La Personnalité des animaux VI, 24) attribue une technique semblable aux renards qui chassent les outardes du Pont : allongés sur le dos, ils attirent les outardes et quand elles sont proches, ils se retournent pour les attaquer.

36 Texte établi et traduit par S. Amigues, CUF, 1993.

37 Voir Xénophon, Cyropédie VII, 5, 11; Strabon, XV, 3, 10; Pline, Histoire naturelle XVI, 223; Plutarque, Propos de table VIII, 4, 5 et Aulu-Gelle, Nuits attiques III, 6, 1-2.

${ }^{38} R P \mathrm{~V}, 3,6$

39 On peut observer la longue postérité de ce bombement victorieux du palmier jusque dans l'Emblematum liber de l'Espagnol A. Alciato, en 1531. L'emblème XXXVI, qui fait du palmier le symbole de la résistance devant l'adversité, représente un homme se tenant par les deux mains à deux branches de palmier : loin de rompre ou de fléchir sous le poids, les palmes, au contraire, soulèvent l'homme du sol. Voir S. SEBASTIAn, Emblemas, éd. et com. de l'Emblematum Liber de A. Alciato (1531), Madrid, 1985.
} 
bois de palmier qui laisse les botanistes et les commentateurs d'aujourd'hui muets $^{40}$ ou circonspects ${ }^{41}$, semble devoir être rangée au nombre des mirabilia $^{42}$. Si ce n'est pas dans la nature que les Grecs ont rencontré ce mouvement aberrant du bois de palmier, c'est dans le regard qu'ils portaient sur cet arbre. Cette étrange «conduite de retournement», les Grecs ont pu la rencontrer dans l'association ancienne entre l'arbre qui a vu naître les Jumeaux de Délos et leurs instruments à bras et à cordes ${ }^{43}$. J'ai montré que les Minoens et les Grecs ont très vraisemblablement utilisé les qualités et les propriétés du palmier, un bois aisément disponible et qu'ils connaissaient bien, pour fabriquer leurs arcs et leurs premières cithares ${ }^{44}$. Or l'arc et la lyre, au sens générique du terme, sont des "puissances de retournement " ${ }^{45}$ capables, comme le palmier-dattier, d'opérer les renversements de puissance propres à la mètis ${ }^{46}$. C'est là, entre l'arc et la lyre, et plus près de l'arc que de la lyre, que j'ai proposé de situer le noyau organisateur des domaines et des façons d'agir d'Apollon, le lieu symbolique où viennent s'ordonner ses différentes facettes. Voilà deux instruments liés par une très étroite parenté en pays grec - comme dans d'autres sociétés séparées ou non des Grecs par le temps et par l'espace - beaucoup moins attributs ou signes de reconnaissance qu'objets constitutifs du dieu, qui par leur matérialité, par les images et les valeurs qu'ils portent ou appellent font «tenir » Apollon debout.

40 Ainsi, P. Munier (Le palmier-dattier, Paris, Maisonneuve \& Larose, 1973), R.A. Brac DE LA PERRIERE (Le palmier-dattier, Tunis, Edisud, 1995) et S. Amigues (o.c. [n. 36], n. 3 à V, 6, 1)

41 STEIER, «Phoinix (1) », RE, XX, 1 (1941), col. 386-403, énumère les références littéraires en reconnaissant qu' " on ne peut pas éclaircir ici la véracité de cette observation ni sur quoi repose cette propriété du bois de Phoinix (...)» («Ob diese Beobachtung richtig ist, und worauf diese Eigenschaft des P.-Holzes etwa beruht, kann hier nicht geklärt werden », col. 393, 1. 49-52). R. MARACHE (n. 4 à Nuits Attiques III, 6, 2, CUF, 1967) y voit une « exagération » et pour J. ANDRÉ (n. 1 à Histoire naturelle XVI, 223, CUF, 1962), il s'agit d'une " propriété faussement attribuée au bois de palmier, due sans doute à un cas fortuit ».

42 Ainsi que le font Plutarque et Aulu-Gelle. Quant à Théophraste, il reprend sans doute un discours répandu sur le palmier-dattier sans pouvoir l'expliquer davantage. Sur l'attitude de Théophraste en face des «accidents » et des «mystères » du monde végétal, je renvoie à S. AMIGUES, "Technologie forestière et travail du bois dans l'Historia plantarum de Théophraste », in J.-P.

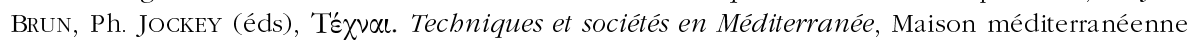
des sciences de l'homme, Maisonneuve et Larose, 2001, p. 149-152.

3 De cette association, Théophraste et ses contemporains ne gardent plus le souvenir. Ainsi, c'est à l'insu de Théophraste que l'arc et la très sagittaire Artémis transpirent par de nombreux pores du texte des Recherches sur les plantes sur le palmier-dattier et la phéniciculture. Voir Ph. Monbrun, «Artémis et le palmier-dattier», Pallas 35 (1989), p. 69-93. Cet article reprend les grandes lignes d'un Mémoire de Maîtrise homonyme soutenu en 1987, à l'Université de ToulouseLe Mirail, sous la direction de P. Briant et Cl. Leduc. Voir encore mon article, "Apollon : de l'arc à la lyre », in Brulé - Vendries, o.c. (n. 4), p. 88-92.

44 Voir les deux articles cités à la note précédente.

45 J'emprunte l'expression à Detienne - Vernant, o.c. (n. 32)., p. 45 : comme la mètis, le renard est une «puissance de retournement ».

46 C'est un des aspects de la quasi-identité de ces instruments que je me suis attaché à démontrer dans la thèse que j’ai soutenue sous la direction de P. Brulé : Les voies sonores d'Apollon : l'arc, la lyre et les oracles, Université de Rennes 2, déc. 2003. 


\section{L'arc « réflexe »}

L'arc, tout d'abord ${ }^{47}$. Il en existe deux types, tous deux portés par Apollon et Artémis. Le premier, l'arc «simple » est fait d'une seule pièce de bois courbée par une corde. Le second, l'arc «composite», est une arme très élaborée et puissante faite de plusieurs matériaux, bois, corne et tendon, qui rendent ses bras beaucoup plus flexibles et résistants. Pour bander un tel arc, c'est-àdire y installer la corde, l'archer doit en inverser la courbure naturelle, celle qu'il a sans la corde quand il est débandé.

Un arc de ce type est appelé « réflexe » ce qui correspond à l'épithète homérique palintonos désignant un arc dont la mise sous tension se fait en renversant vers l'arrière sa position initiale de la position au repos - sans la corde - vers la position bandée - avec la corde - puis la position armée, quand l'arc est prêt à tirer (Fig. 6, ci-contre).

En sens inverse, quand il passe de l'état armé à l'état de repos, cet arc connaît un autre retournement, rendu par l'épithète palintropos dans le fragment 51 d'Héraclite, et qui signifie « qui se retourne, qui revient sur soi-même ». Il y a là deux retournements de courbure qui caractérisent le fonctionnement de l'arc réflexe : le premier permet à l'arc d'emmagasiner beaucoup d'énergie et le second lui permet de la libérer avec une force inégalée. Aussi, dans les mains de l'archer, l'arc palintone et palintrope ne se contente-t-il pas de renverser sa position. Il est aussi capable de « retourner» la situation au profit de celui qui le porte. Sur le champ de bataille, notamment, l'arc permet, même au plus
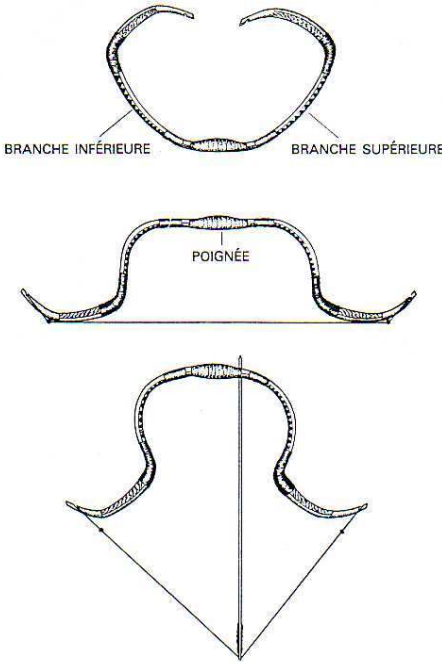
faible ou au plus lâche, de triompher, de loin et sans risque, d'un guerrier plus fort ou plus brave sur lequel il ne l'aurait pas emporté au corps à corps. De là le mépris qui frappe l'archer et son arme, depuis Homère jusqu'à la Guerre du Péloponnèse et la controverse de l'Héraclès d'Euripide sur les qualités et les défauts respectifs de l'hoplite et de l'archer ${ }^{48}$. L'affrontement entre Pâris-Alexandre et Diomède, dans l'Iliade, est à ce propos des plus significatifs : l'archer lâche et efféminé met hors de combat le seul, avec Ajax, à talonner Achille pour la valeur guerrière ${ }^{49}$. Quel écart aussi entre ce même

47 Je condense ici en quelques lignes des développements consacrés dans ma thèse à l'archerie grecque en général et apollinienne en particulier. Dans l'attente d'une prochaine publication, je renvoie le lecteur aux pages 62-69 et 79-80, notamment, de l'article « Apollon : de l'arc à la lyre », l.c. (n. 43).

48 Euripide, Héraclès, 157-205.

49 Iliade XI, 373-395. 
Pâris, qu'on pourrait appeler «le pire des Troyens », et Achille, «le meilleur des Achéens" (aristos Akhaiôn) ${ }^{50}$, qu'il tue pourtant de sa flèche. Un événement marquant de la Guerre du Péloponnèse, comme l'affaire de Pylos, en 425 , montre bien que l'arc ne retourne pas seulement l'issue la plus logique et la plus attendue du combat, mais renverse aussi les codes et les valeurs de la guerre hoplitique ${ }^{51}$. Le courage de l'hoplite qui se bat «selon les règles », qui garde le rang pour soutenir le choc des lances ennemies, est impuissant face à cette mort qu'il ne voit pas venir et qui frappe aveuglément sans faire de différence « qualitative» entre ses victimes ${ }^{52}$.

\section{Chelys et kitbara}

L'instrument de musique apollinien est aussi une "puissance de retournement» très proche de l'arc. Manipulée par Apollon dans la joute qui l'oppose à Marsyas, la cithare tient à la fois de la palintonie et de la palintropie de l'arc réflexe. Comme l'archer qui renverse la courbure initiale de l'arc palintonos pour le bander et le rendre apte au tir, Apollon inverse la position initiale de la cithare pour pouvoir en jouer. Comme les bras de l'arc palintropos qui, après le tir, voient à nouveau leur courbure inversée et reviennent à leur position naturelle quand l'arc est débandé, la cithare voit, après le jeu, sa position à nouveau renversée sur elle-même et ses bras retrouver leur orientation première. La gestuelle citharistique que maitrise Apollon dans ce duel musical s'apparente à celle que doit maitriser l'archer pour utiliser un arc réflexe $^{53}$. Cette même gestuelle invite au rapprochement avec le comportement du palmier-dattier si cher au dieu : la cithare dont Apollon peut jouer anapalin, «en sens contraire» des autres instruments et qui apporte ainsi la victoire, évoque le bois de palmier-dattier qui, selon Théophraste, se courbe anapalin, « en sens contraire » des autres bois, ce qui lui permet de triompher des plus lourdes charges.

Nous avons vu comment la cithare, en renversant sa position, renverse en même temps la situation en permettant à Apollon de l'emporter sur Marsyas qui avait la prétention d'être meilleur musicien que lui. L'Hymne homérique à Hermès fait aussi de la lyre un instrument né sous le signe du retournement et de la tortue qui lui a donné son nom, chelys. Pareillement, les auteurs anglosaxons parlent de "tortoise ». Hermès, le premier lyropoios, ou "facteur de lyre », commence par retourner la tortue qu'il a choisie, par la mettre sur le

50 Iliade I, 244, 412 et XIV, 271, 274. Voir aussi IX, 110. Sur ce titre décerné à Achille, je renvoie à G. NAGY, Le meilleur des Achéens. La fabrique du béros dans la poésie grecque archaïque, Paris, Seuil, 1994, p. 49-65, principalement.

51 Thucydide, IV, 32-38 et 40, 1-2.

52 Voir, par exemple, Plutarque, Apophtegmes laconiens, 46 : l'amertume se lit dans les dernières paroles prêtées à un Spartiate tué par une flèche.

53 Voir Ph. Monbrun, «Les gestes de l'archer et du cithariste : des « corps à corps » apolliniens où l'esprit prend le relais », colloque international Gestuelles, Attitudes, Regards. L'expression des corps dans l'imagerie antique, organisé sous la tutelle du RUOA (Réseau des Universités de l'Ouest Atlantique), Rennes, 23 avril 2004, à paraître aux Presses Universitaires de Rennes. 
$\operatorname{dos}^{54}$ : il s'agit de retirer la partie ventrale de la carapace, le «plastron », pour pouvoir vider l'animal et obtenir la caisse de résonance de l'instrument. Le discours bienveillant et louangeur que le petit luthier adresse à la tortue montre également que ce retournement qui la tue est aussi un retournement de fortune pour un animal dédaigné de son vivant et qui, après sa mort, se voit accorder, sous les traits de la lyre, tout à la fois une seconde existence et la haute considération des hommes ${ }^{55}$. Mais la lyre a de qui tenir! La «tortoise» est redevable à la tortue dont elle est issue. La fable 352 d'Ésope qui inspira La Fontaine, raconte comment cet animal à la démarche traînante et qui porte sa carapace comme une charge, est plus rapide que le lièvre, « retournant» ainsi une course qui semblait perdue d'avance. La tortue devenue lyre, c'est l'instrument de musique qui montre sa puissance de retournement. Après le vol du troupeau d'Apollon par Hermès, la lyre permet au plus petit et au plus faible des deux protagonistes de se tirer, à très bon compte, d'un mauvais pas. Hermès nouveau-né, d'abord par les seules notes de sa lyre, puis chantant en s'accompagnant de l'instrument, « retourne » Apollon, transformant la colère du frère aîné en admiration et en amitié à son égard ${ }^{56}$. Cl. Leduc a mis en évidence le rôle de la lyre dans l'instauration de la philotès (v. 507), à la fois «lien d'amitié et de respect mutuel» entre les deux frères ${ }^{57}$ : à l'issue d'une véritable transaction commerciale, la lyre est le paiement qu'Hermès contraint Apollon d'accepter pour le prix des vaches qu'il lui a volées. Hermès revient de loin et, grâce à sa lyre, c'est aussi sa propre situation qui s'est « retournée » de façon spectaculaire : de nouveau né d'un jour à peine sorti de ses langes, reconnu ni par les Olympiens, ni par les hommes et passible de la vengeance d'Apollon, il passe à l'état de dieu de plein droit, pourvu des attributs de sa puissance et des honneurs dont il est redevable.

Dans les mains d'Orphée aussi, la lyre est une "puissance de retournement ». Ainsi, dans les Argonautiques d'Apollonios de Rhodes, Orphée, qui est le plus faible des Argonautes, parvient pourtant, grâce à son chant et aux accents de sa phorminx, à charmer l'équipage de l'Argo et à calmer les esprits échauffés par une dispute ${ }^{58}$. Plus tard, sa virtuosité instrumentale lui permet de l'emporter sur les dangereuses Sirènes : en brouillant leur chant, la musique de la phorminx sauve les Argonautes d'une mort certaine ${ }^{59}$. Dans les Images, Philostrate le Jeune décrit un tableau qui représente Orphée citharode envoûtant des animaux sauvages, prédateurs et proies se côtoyant sans dommage $^{60}$. Enfin, Orphée parvient à charmer Charon, Cerbère, Perséphone et Hadès lui-même dont il obtient de pouvoir accéder au monde du dessous

54 Hymne homérique à Hermès, 41-42

55 Ibidem, 30-38.

Ibid., 416-462.

$57 \mathrm{Cl}$. LEDUC, "Cinquante vaches pour une lyre! Musique, échange et théologie dans

l'Hymne à Hermès I », in BRulé - VENDRIES, o.c. (n. 4), p. 19-36.

58 Argonautiques I, 494-515 : la musique adoucit les mours.

59 Argon. IV, 902-919.

60 Philostrate le Jeune, Images, 6, 1-3. 
pour en ramener les morts qu'il est allé y chercher et notamment son épouse Eurydice $^{61}$. La musique de l'instrument à cordes, accompagnant ou pas le chant, réussit à « inverser » la nature profonde de ceux qui les écoutent. C'est finalement la mort, suprême "retournement», qui se laisse fléchir sous l'emprise du pouvoir qu'Orphée tire de sa lyre et de son chant. Ces réflexions m'amènent à considérer avec intérêt la lecture que J. Svenbro, fait de l'agôn musical entre Apollon et Marsyas, tel qu'il est raconté par le pseudoApollodore : en se fondant sur l'analyse des rapports antithétiques entre la chelys née de la tortue et la pierre tombale, il propose de voir dans la première, l'instrument efficace du retour de la mort muette à la vie sonore, et dans la seconde, le mouvement contraire et l'impossibilité de ce retour. Tenue à l'endroit, les bras pointés vers le haut, la lyre, et celle d'Orphée tout spécialement, serait l'instrument de retour du défunt vers le monde des vivants, l'instrument capable de vaincre la mort, alors qu'au contraire, la lyre d'Apollon inversée vers le sol, serait le "signe funéraire » qui condamne Marsyas à mort et l'entraîne sans espoir de retour vers le monde infernal ${ }^{62}$.

D'une position à l'autre, la lyre changerait donc de signe. L'arc n'est-il pas aussi dans ce cas ? Je suis frappé de la symétrie entre la nature ambivalente de la lyre qui se dessine ici et le statut ambigu de l'arc tel que l'ont défini J. Le Goff et P. Vidal-Naquet : médiéval ou grec, «l'arc est un signe dont la valeur n'est donnée que par la position qu'il occupe dans le système » ${ }^{63}$. En fonction du contexte dans lequel il est utilisé et du statut de celui qui le porte, il pourra être "signe de chute ou signe de remontée " ${ }^{64}$, au point que P. VidalNaquet a pu parler, pour le monde grec comme pour le monde médiéval, d'un «arc-plus » et d'un «arc-moins » ${ }^{65}$. Il en va de même pour la lyre : en fonction de la position qu'elle occupe - dans l'espace, ici -, tenue vers le haut ou retournée vers le bas, la lyre est, au sens propre comme au sens figuré, «signe de remontée » de la mort vers la vie - c'est la lyre d'Orphée qui ramène Eurydice -, ou «signe de chute » de la vie vers la mort - c'est la lyre d'Apollon qui précipite Marsyas aux Enfers.

${ }^{61}$ Cf. Euripide, Alceste, 357-362; Isocrate, Busiris, 8; Diodore, IV, 25 et le ps.-Apollodore, I, 3 , 2. Dans les Géorgiques (IV, 485-503),Virgile raconte comment Orphée, près de réussir, perd une seconde fois Eurydice parce qu'il s'est retourné vers elle alors qu'ils remontaient vers la lumière, rompant ainsi le pacte passé avec Hadès. Le même échec est décrit par Ovide au Livre X des Métamorphoses (v. 50-63). Pour la tradition pré-virgilienne, au contraire, l'anabase d'Orphée et de sa défunte épouse vers le monde des vivants est une réussite. Seul Platon, dans le Banquet (179 d), présente Orphée sous un jour défavorable puisqu'il n'obtient pas de ramener Eurydice à la vie. Voir J. Heurgon, «Orphée et Eurydice avant Virgile », Mélanges d'Archéologie et d'Histoire de l'École française de Rome (1932), p. 5-60.

J. SvenBRO, "'Ton luth, à quoi bon ?' La lyre et la pierre tombale dans la pensée grecque ", Mètis 7 (1992), p. 144, 148 et 158-159.

63 J. LE GOFF, P. VIDAL-NAQUET, « Lévi-Strauss en Brocéliande. Esquisse pour une analyse d'un roman courtois », in R. Bellour, C. CléMENT (éds), Lévi-Strauss, Paris, Gallimard, 1979, p. 275.

64 Ibidem, p. 277.

65 P. VIDAL-NAQUET, Le chasseur noir. Formes de pensée et formes de société dans le monde grec, Paris, La Découverte / Maspéro, 1983 (coll. «Fondations »), n. 49, p. 193. 


\section{Du retournement de la cithare au retournement de la peau}

Un dernier point reste en suspens, c'est la peau écorchée de Marsyas ${ }^{66}$ accrochée à son arbre. L'analyse de la notion de retournement et l'exploitation que j'en ai faite pour comprendre l'épisode de Marsyas, m'amènent à me demander s'il ne faut pas comprendre de la même manière l'écorchement qu'Apollon fait subir au satyre : après l'avoir vaincu en retournant sa cithare, il le punit en lui retournant la peau ${ }^{67}$. De nombreux textes, en effet, laissent entendre que Marsyas n'a pas été écorché une lanière de peau après l'autre, mais par une sorte de retournement de sa dépouille tout entière, retroussée à la manière d'une outre. C'est ainsi qu'Hérodote (VII, 26) raconte comment, en 481, l'armée de Xerxès, en route pour Sardes,

... pénétra en Phrygie et, marchant à travers ce pays, parvint à Kélainai, où sont les sources du Méandre et celles d'un autre fleuve non moins important que le Méandre, qui a nom Catarractès; il jaillit au milieu même de la place publique de Kélainai et se jette dans le Méandre. Là aussi [dans l'agora de Kélainai] est suspen-

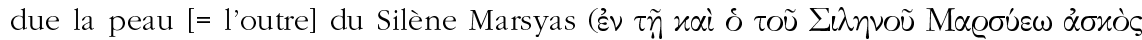

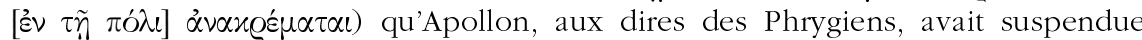

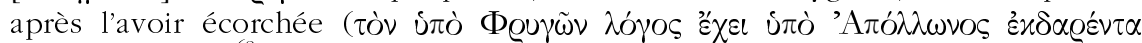
$\dot{\alpha} \nu \alpha \varkappa \varrho \varepsilon \mu \alpha \sigma \theta \tilde{\eta} \nu \alpha)^{68}$.

Dans l'Euthydème de Platon, Ctésippe fait à Socrate, sur le mode plaisant, la réponse suivante $(285 \mathrm{c}-\mathrm{d})$ :

Moi aussi, Socrate, je suis prêt à me remettre aux mains des étrangers, même s'ils veulent m'écorcher encore plus qu'ils ne le font en ce moment, à condition

66 B. Sergent m'a signalé que le nom même de "Marsyas » - qui n'a pas d'étymologie grecque - est lié à l'écorchement du satyre. Voir G. DumÉzil, Apollon sonore et autres essais. Esquisses de mythologie, Paris, Gallimard, 1982, p. 49: alors qu'il évoque les influences des civilisations proche-orientales voisines sur la musique grecque et Apollon musicien, dans la quatrième Esquisse de la "Suite vocale et apollinienne", le comparatiste donne comme étymologie pour "Marsyas », "arménien mort 'peau', mort'el 'écorcher', mort'uadz 'écorché' ». Dans Le livre des dieux. Celtes et Grecs II (Paris, Payot et Rivages, 2004, p. 349), B. Sergent y voit l'étymologie « la plus solide » et renvoie à Hérodote, VII, 73, selon lequel les Arméniens sont une colonie des Phrygiens : voilà qui corrobore une étymologie arménienne pour le nom d'un personnage originaire, pour toute la tradition grecque, de Phrygie. L'auteur revient à plusieurs reprises sur le mythe et l'écorchement de Marsyas. Voir en particulier, pages 348-351, l'hypothèse d'un mythe remontant à « un état archaïque de la mythologie d'Apollon » dans lequel le dieu aurait été supplicié à un arbre, peut-être même écorché; mythe « refoulé » ensuite, puis transposé sur un personnage mythique du centre anatolien intégré dans la mythologie grecque comme partenaire d'Apollon. Voir aussi n. 391, p. 681-2 et p. 447-448 : l'écorchement est une pratique indo-européenne anciennement attestée, ainsi dans le monde iranien et en Grèce.

${ }_{68}^{67}$ Je remercie V. Pirenne-Delforge de m'avoir suggéré ce séduisant parallèle.

68 Texte établi et traduit par Ph. E. LEGRAND, CUF, 1951. J'ai modifié la traduction de la dernière phrase pour rester au plus près du texte grec. Relatant, dans l'Anabase (I, 2, 7-9), l'étape que fit Cyrus le Jeune en 401, pour faire la revue de ses troupes à l'intérieur du vaste paradis de Kélainai, Xénophon évoque aussi les gloires topiques : "C'est là, dit-on, qu'Apollon vainqueur de

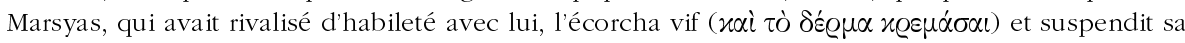
peau dans la grotte où sont les sources de la rivière ( elle s'appelle le Marsyas. » Texte établi et traduit par P. MASQuERAY, CUF, 1959. 


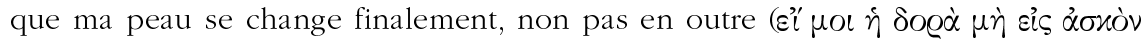

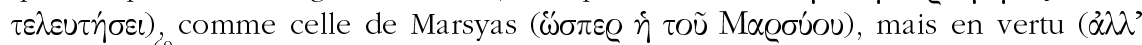

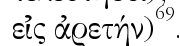

Dans ses Images, Philostrate le Jeune décrit une peinture représentant l'agôn entre Apollon et Marsyas alors que le dieu termine sa prestation et que le satyre se sait déjà perdu $(2,1)$ :

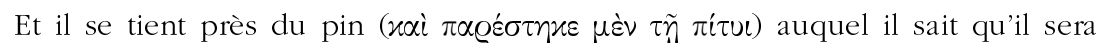

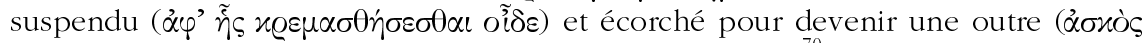
$\delta \varepsilon \delta \alpha \dot{Q} \theta \alpha$ ), ayant lui-même prononcé cette peine contre lui ${ }^{70}$.

Quant à Nonnos de Panopolis, il évoque en ces termes le châtiment de l'insolent Marsyas, dans Les Dionysiaques (XIX, 319-22) :

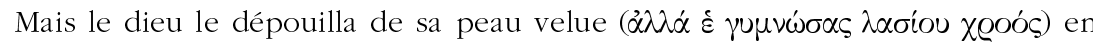

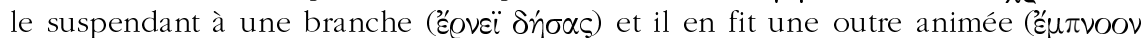

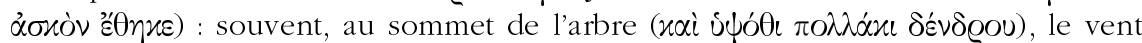

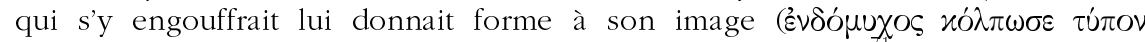

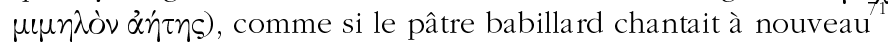

Ces quatre textes désignent la dépouille de Marsyas par le terme «askos », pour lequel P. Chantraine donne «'peau d'un animal écorché', d'où usuellement 'outre' qui en est faite, notamment pour contenir du vin ou comme soufflet $»^{72}$. Ce terme suggère que la peau écorchée garde, comme une outre, la silhouette du corps du satyre, ce que confirme Nonnos quand il précise que le vent redonnait en quelque sorte figure humaine au corps de Marsyas en gonflant sa peau ${ }^{73}$. L'épigramme funéraire VII, 696 qu'Archias de Mytilène adresse à Marsyas invite à la même lecture : «Tu te balances, ton corps de

69 Texte établi et traduit par L. MERIDIER, CUF, 1949.

70 Je propose ici ma traduction. Le texte grec est celui sur lequel s'appuie la traduction d'A. FAIRBANKS, $L C L, 1931$

${ }^{71}$ Texte établi et traduit par J. Gerbeau, Fr. VIAN, CUF, 1992.

72 P. Chantraine, s.v. ג̇oxós, Dictionnaire étymologique de la langue grecque, Paris, Klincksieck, 1968. Cf. LSJ, s.v. : "skin, hide, (...); but usually, skin made into a bag, esp. wineskin. » Voir Fr. LISSARRAgue, Un flot d'images. Une esthétique du banquet grec, Paris, A. Biro, 1987, p. 6673 : les vases à boire athéniens des $\mathrm{VI}^{\mathrm{e}}$ et $\mathrm{V}^{\mathrm{e}}$ siècles montrent les jeux de l'outre auxquels, dans le cadre du symposion, se livrent éphèbes et satyres en hommage à Dionysos.

73 L'outre remplie d'air qu'est devenu l'aulète Marsyas et le sens de «soufflet » relevé par P. Chantraine, invitent au rapprochement avec le sac de peau qui sert de réservoir d'air à la cornemuse. Dion Chrysostome (Discours, LXXI, 9) cite l'askos de la cornemuse, instrument que Néron préfère à l'aulos parce qu'il ne déforme pas son visage quand il en joue. Martial (X, 3, 8) donne le nom grec du cornemuseur, l'ascaules (= $\alpha \sigma \varkappa \alpha u ́ \lambda \eta \varsigma)$, appelé de la même façon utricularius en latin (cf. Suétone, Néron, 54), terme dérivé d'utriculus, «petite outre ». Sur la place de cet instrument à vent dans la musique hellénistique et romaine, je renvoie à M.L. WEST, Ancient Greek Music, Oxford, Clarendon Press, 1992, p. 107-109, ainsi qu'à A. BAUDOT, Musiciens romains de l'Antiquité, Paris, Klincksiek, Presses de l'Université de Montréal, 1973, p. 71. Sur le rapprochement entre l'outre Marsyas et l'outre de la cornemuse, voir aussi LECLERCQ-NEVEU, l.c. (n. 14), p. 265. 


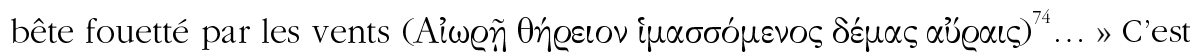
la silhouette même de Marsyas qui ballotte au gré du vent tout comme c'est elle que les accents de l'aulos semblent rendre à la vie, dans l'Histoire variée d'Élien (XIII, 21) :

À Kélainai, si quelqu'un joue de l'aulos sur le mode phrygien près de la peau

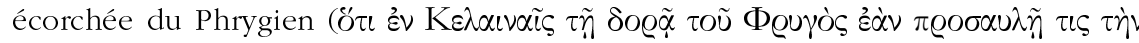

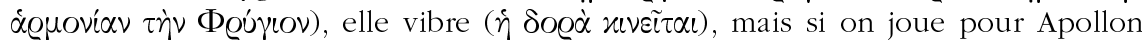

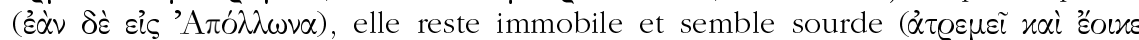
$x(\omega) \varphi \tilde{n})^{75}$.

Cette dépouille animée par le vent et sensible par delà la mort à la musique de l'aulos, ne peut se concevoir, à l'image d'une outre, que comme une enveloppe, une espèce de mannequin qui a l'apparence du vivant ${ }^{76}$. Une fois Marsyas suspendu à l'arbre de son supplice - un pin, le plus souvent ${ }^{77}$, la peau ne lui est donc pas retirée lambeau après lambeau mais d'un seul tenant, et il n'y a guère là qu'une seule technique possible: un véritable retroussement de la peau du haut jusqu'au bas du corps, comme on peut encore le voir faire sur les lapins dans les campagnes, ou comme je l'ai vu pratiquer sur des chèvres et des boucs par des bergers crétois. Cette peau ainsi retroussée permet de mieux saisir la pertinence de la comparaison avec l'askos: l'outre elle-même est une peau retournée, la surface extérieure étant constituée par la peau qui se trouvait côté chairs et la surface intérieure, celle en contact avec le liquide, par ce qui était l'extérieur de la peau. Un tel procédé évoque la très déconcertante manœuvre de retournement prêtée par les Grecs à un poisson qu'ils nommaient de façon significative «renard».

${ }^{74}$ Anthologie Palatine VII. Epigrammes Funéraires. Texte établi par P. Waltz et traduit par Ed. Des Places, M. Dimitrescu, H. Le Maitre et G. Soury, CUF, 1941

75 Traduction légèrement modifiée d'A. LuKINOVICH et d'A.-F. MORAND, Paris, Les Belles Lettres, 1991 (La Roue à Livres). Le texte grec est celui sur lequel s'appuie la traduction de N.G. Wilson, LCL, 1997.

76 M. Patera a porté à ma connaissance deux gravures d'écorchés tirées de Quatre siècles de surréalisme, l'art fantastique dans la gravure, préf. de M. Brion, Belfond, 1973. La planche 53, extraite de l'Anatomia del corpe humano de J. Valverde, Rome, 1560, montre un écorché qui «a ceci d'étonnant, qu'il tient d'une main le couteau qui l'a écorché, de l'autre, sa peau qui pend comme une défroque vide et la place des yeux et de la bouche dessine un masque tragique, c'est pourquoi on a quelquefois intitulé cette planche 'L'Homme au masque'» (p. 197). Il me semble que cette peau d'un seul tenant, avec ses quatre membres bien distincts, évoque la silhouette pendante de Marsyas et ce d'autant plus que les traits nettement faunesques et la longue barbe suggèrent, de la part de l'auteur, la volonté d'évoquer le mythe de Marsyas au lecteur humaniste de la Renaissance. Voir aussi la planche 123, extraite du frontispice de L'Anatomia reformata de Th. Bartholin, Leyde, 1651, et son commentaire p. 208.

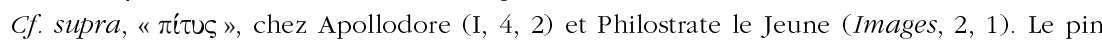
de la tradition est un platane chez Pline, Histoire naturelle, XVI, 240. Sur l'arbre porteur de la dépouille de Marsyas, voir Ch. VENDRIEs, «Des plantes et des dieux. Mythes, cultes et musiques dans l'Antiquité gréco-romaine ", L’homme, le végétal et la musique, FAMDT Editions, 1996 (coll. «Modal»), p. 92-93. Le pin paraît d'autant plus approprié pour le châtiment sanguinolent de Marsyas que - le fait a été rappelé par $\mathrm{Cl}$. Leduc dans la discussion qui suivit cette communication - son bois est rouge quand il a perdu son écorce. 
Ainsi Plutarque, quand il décrit le moyen de se débarrasser de l'hameçon, dans son traité Sur l'intelligence des animaux (977b) :

Le renard ( $(\alpha \lambda \omega \dot{\omega} \eta \xi)$ n'approche pas souvent l'appât mais il fuit la ruse; s'il est pris, il s'en débarrasse. En effet, grâce à sa souplesse et à sa flexibilité, il a une aptitude naturelle à transformer son corps ( $\pi \varepsilon \dot{\varphi} \varphi x \varepsilon \ldots \mu \varepsilon \tau \alpha \beta \dot{\alpha} \lambda \lambda \varepsilon \iota \nu \tau o ̀ ~ \sigma \tilde{\omega} \mu \alpha)$ et à le

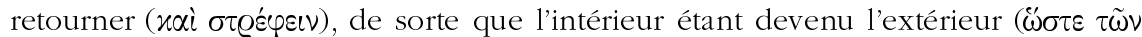

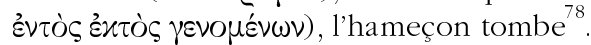

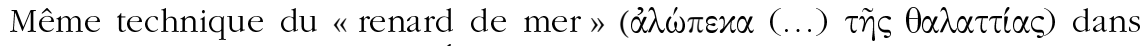
La Personnalité des animaux d'Élien (IX, 12) :

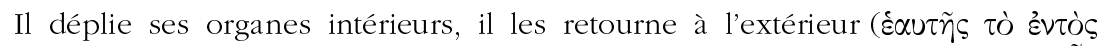

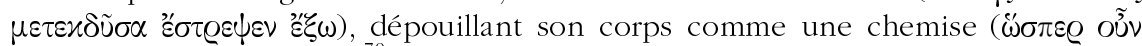

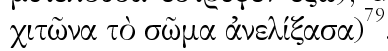

Dans cette astuce proprement «renversante » du «poisson-renard " qui retourne l'intérieur de son corps vers l'extérieur, M. Detienne et J.-P. Vernant reconnaissent le « cas limite du renversement » : pour expulser l'hameçon, ce poisson se retrousse, "se retourne comme un gant». Sous le signe d'une mètis très palintrope, qui aime tout ce «qui se retourne », ce " qui revient sur soi-même », l'air de famille me paraît frappant entre la victorieuse manœuvre de cet animal qui est capable de se retrousser pour l'emporter sur le pêcheur et le châtiment d'Apollon qui manifeste sa victoire en retroussant la peau de Marsyas. Je pousserais volontiers la parenté avec le double renversement de courbure qui donne toute sa puissance à l'instrument réflexe d'Apollon (Fig. 6) : les deux bras qui basculent autour de la poignée de l'arc créent l'illusion qu'il se retrousse lui aussi « comme un gant» de part et d'autre de la seule partie immobile de l'instrument.

Ainsi, la version de l'agôn musical entre Apollon et Marsyas que donne le ps.-Apollodore n'a rien d'anecdotique ni de fortuit. Le retournement de la lyre par le dieu musicien, mais aussi le retournement de la peau du vaincu, s'insèrent dans un complexe apollinien articulé autour des "conduites de retournement » que connaissent les deux instruments à bras et à cordes d'Apollon et l'arbre de la «nativité » délienne. Renversements de l'arc palintone et palintrope, retournements de la lyre, courbure anapalin du palmier-dattier, retroussement de la peau de Marsyas, autant de conduites qui mettent en jeu

\footnotetext{
${ }^{78} \mathrm{Je}$ propose ma traduction. Le texte grec est celui sur lequel s'appuie la traduction de H. Cherniss et W. C. Helmbold, LCL, 1957.

J'ai retenu la traduction de Detienne - Vernant (o.c. [n. 32], p. 44). A. ZuCKer (Paris, Les Belles Lettres, 2001 [La Roue à Livres]) traduit : «Il (...) dévagine ses entrailles à l'extérieur comme s'il se déshabillait, en retournant son corps comme un gant (...). » Dans l'Histoire des animaux (IX, 37, 621a 6 sq.), Aristote attribue la même manœuvre de retournement à la scolopendre de

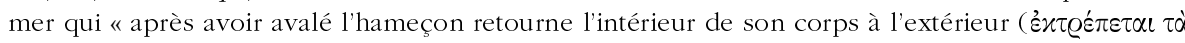

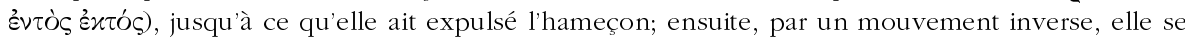

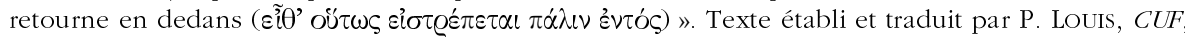
1969. On trouve, dans l'Histoire naturelle de Pline (IX, 145), un récit semblable imité d'Aristote.

80 DETIENNE - VERNANT, o.c. (n. 32), p. 44.
} 
à la fois un renversement de position et un retournement de situation. Il y a là, sous le signe de la mètis, une saisissante communauté de caractères.

Philippe MONBRUN

Crescam - Université de Rennes 2

6, av. Gaston Berger

F - 35043 RENNES Cedex

\section{Légendes des illustrations}

Figure 1. Marsyas vaincu. Cratère en cloche campanien, vers 360-330. Copenhague, Mus. Nat. 3757. Dessin de l'auteur d'après le LIMC, «Artémis », II, 2 (1984), fig. 1429, p. 562

Figure 2. Marsyas condamné. Médaillon d'applique gallo-romain signé » Felix», seconde moitié du i ${ }^{\text {er }}$ siècle ap. J.-C., A. Audin, H. Vernet, Gallia 30 (1972), p. 235-246, fig. 1.2. Dessin de l'auteur d'après le LIMC, «Apollon / Apollo », II, 1 (1984), fig. 591, p. 456.

Figure 3. Apollon vainqueur joue de la lyre retournée. Plaque de grès en relief provenant d'un monument funéraire romain, de Bierbach, en Allemagne. Speyer, Mus. Hist. du Palatinat. Dessin de l'auteur d'après le LIMC, II, 2, «Apollon / Apollo» (1984), fig. 584, p. 351.

Figures 4. Aplu tient la lyre à l'envers. Fragment provenant d'un groupe de statuettes votives étrusques en terre cuite, de Véies ( $c a 400$ exemplaires) et Faléries. Véies, sanctuaire du Portonaccio, v ${ }^{e}$ siècle. Rome, Villa Giulia. Dessin de l'auteur d'après le LIMC, II, 2, « Apollon / Aplu» (1984), fig. 89, p. 294.

Figure 5. Satyre aulète couché. Amphore attique FR, peintre de Berlin, vers 490. Paris, Musée du Louvre G 201. D'après Fr. LissarRague, Un flot d'images. Une esthétique du banquet grec, Paris, éd. A. Biro, 1987, fig. 24, p. 42.

Figure 6. Les trois positions de l'arc palintone et palintrope (au repos ou débandé; encordé ou bandé; armé, prêt à tirer). D’après E. Mc EwEn, R. Miller et Ch. BERGMAN, «La conception et la fabrication des arcs anciens ", Pour la science (éd. française de Scientific American) 166, août 1991, p. 77. 